\title{
Effektivität und Praktikabilität einer internetbasierten Asthma-Nachschulung im Kindes- und Jugendalter
}

\author{
Effectiveness and Practicality of an Internet-based Asthma Refresher Course \\ for Children and Adolescents
}

Autoren

Institute
A. Schmidt ${ }^{1, *}$, T. Greuter ${ }^{2, *}$, A. Möller², J. O. Steiß ${ }^{1}$

Zentrum für Kinderheilkunde und Jugendmedizin, Pädiatrische Pneumologie und Allergologie, Justus-Liebig-Universität Gießen

Klinik für pädiatrische Pneumologie, Universitäts-Kinderkliniken Zürich eingereicht 19.8 .2013

akzeptiert $\quad 20.10 .2013$

\section{Bibliografie \\ DOI http://dx.doi.org/ \\ 10.1055/s-0033-1358921 \\ Online-Publikation: 3.12.2013 \\ Pneumologie 2014; 68: 259-265 \\ (c) Georg Thieme Verlag KG \\ Stuttgart · New York \\ ISSN 0934-8387}

\section{Korrespondenzadresse}

PD Dr. med. Jens-Oliver Steiß

Zentrum für Kinderheilkunde und Jugendmedizin

Abteilung Allgemeine Pädiatrie und Neonatologie

(Leiter: Prof. Dr. K. P. Zimmer)

Pädiatrische Pneumologie und Allergologie

Justus-Liebig-Universität Gießen

Feulgenstraße 12

35385 Gießen

Jens-Oliver.Steiss@paediat.med. uni-giessen.de

\section{Zusammenfassung \\ $\nabla$}

Hintergrund und Methodik: Die Effektivität und Praktikabilität einer strukturierten, internetbasierten Asthma-Nachschulung „Luftikids“ (www. luftikids.de) wurde an 53 Patienten (Alter 8-14 Jahre) im Rahmen einer Pilotstudie evaluiert. Alle Patienten hatten bereits zuvor an einer ambulanten und/oder stationären Asthmaschulung teilgenommen. Es wurde in einer prospektiven Studie der Einfluss einer 4-wöchigen Nachschulung auf Parameter wie Asthmasymptome (Husten, Dyspnoe), Anzahl der ungeplanten Arztbesuche, Gebrauch der Bedarfsmedikation, Anzahl der Schulfehltage, Asthmakontrolle mittels des Asthma Control Questionnaires (ACQ) und Lungenfunktion untersucht. Anhand der Dauer der Programmnutzung und der Anzahl der Logins wurde die Akzeptanz des Spieles untersucht. Die Daten wurden zu Beginn und vier bis sechs Monate nach Beendigung der Online-Nachschulung erhoben.

Ergebnisse: Es konnten signifikante Änderungen bezüglich der Verminderung der Intensität von Asthmasymptomen wie Husten $(p=0,001)$ und Dyspnoe $(p=0,007)$, der Reduktion ungeplanter Arztbesuche $(p=0,005)$, des Gebrauchs der Bedarfsmedikation $(4,0 \pm 6,5$ vs. $1,5 \pm 4,9, \mathrm{p}=<0,002)$ und der Anzahl der Schulfehltage (1,2 $\pm 2,0$ vs. 0,4 $\pm 1,2, p=0,003)$ nachgewiesen werden. Keine Veränderungen waren bei den Lungenfunktionsparametern zu erkennen. „Luftikids“ wurde durchschnittlich 302,5 $\pm 210,9$ Minuten genutzt, die Anzahl der Logins betrug $14 \pm 9$. Die Kinder und Jugendlichen profitierten nach Eigeneinschätzung viel bis sehr viel. Nur 7,5\% gaben keinen Effekt an. Schlussfolgerungen: Die internetbasierte Asthma-Nachschulung „Luftikids“ führte zur verminderten Asthmasymptomatik, Reduktion unge-

\footnotetext{
* gleichermaßen beigetragen
}

\section{Abstract \\ $\nabla$}

Background and Methods: The effectiveness and practicality of the "Luftikids" (www.luftikids.de) structured, internet-based asthma refresher course was evaluated in a pilot study with 53 patients (ages 8-14 years). All patients had previously participated in either an inpatient or outpatient asthma education program. This prospective study examined the effect of a 4-week refresher course on parameters such as asthma symptoms (coughing, dyspnea), the number of unscheduled doctor's visits, use of on-demand medications, number of days absent from school, and asthma monitoring using the Asthma Control Questionnaire (ACQ) and lung function tests. The duration of program use and the number of logins was used to investigate acceptance of the game format. Data were collected at the beginning of the study and at 4-6 months after the end of the online refresher course.

Results: Significant changes were shown with regard to the decrease in intensity of asthma symptoms such as coughing $(\mathrm{p}=0.001)$ and dyspnea $(p=0.007)$, reduction in the number of unscheduled doctor's visits $(p=0.005)$, the use of on-demand medications ( $4.0 \pm 6.5$ vs. $1.5 \pm 4.9$, $\mathrm{p}=<0.002$ ), and the number of days absent from school ( $1.2 \pm 2.0$ vs. $0.4 \pm 1.2, p=0.003)$. No changes in lung function parameters were observed. The "Luftikids" program was used for an average of $302.5 \pm 210.9$ minutes, and the number of logins was $14 \pm 9$. From their self-evaluations, the children and adolescents derived "much" to "very much" benefit. Only $7.5 \%$ reported no effect.

Conclusions: Participation in the "Luftikids" internet-based asthma refresher course resulted in fewer asthma symptoms, a decrease in unscheduled doctor's visits, reduction in the use of on-demand medications, decrease in the number of days absent from school, and improved asthma knowledge. No effect in lung function could be 
planter Arztbesuche, Reduktion der Bedarfsmedikation, Verminderung der Schulfehltage sowie zu einem besserem Asthmawissen. Ein Einfluss auf die Lungenfunktion konnte nicht nachgewiesen werden. Die Ergebnisse sprechen für eine Effizienz und gute Akzeptanz ambulanter internetbasierter Asthma-Nachschulungen. Insbesondere jugendliche Asthmapatienten werden durch diese Form der Nachschulung erreicht und motiviert.

\section{Einleitung \\ $\nabla$}

Das Asthma bronchiale zählt mit einer Prävalenz von 10\% zu den häufigsten chronischen Erkrankungen im Kindes- und Jugendalter. Dabei handelt es sich um eine entzündliche Erkrankung der unteren Atemwege, die durch eine bronchiale Hyperreaktivität sowie variable Atemwegsobstruktion charakterisiert ist. Nicht selten werden in Abhängigkeit von der Schwere der Symptomatik eine verminderte Lebensqualität, Reduktion sozialer Kontakte, geringere physische Aktivität und schulische Leistung im Vergleich mit gesunden Altersgenossen beschrieben [1,2].

Der Nutzen von Asthmaschulungsprogrammen ist unbestritten und wurde in deutschsprachigen und internationalen Studien nachgewiesen [3]. Vorrangiges Ziel ist der Umgang mit der Krankheit und deren Folgen, eine psychologisch-pädagogische Intervention sowie eine Stärkung der Eigenverantwortung und Kompetenz. Neben der Wahrnehmung für den eigenen Körper und dem Verhalten bei Belastungen sollen Möglichkeiten und Anregungen zur Selbsthilfe geschult werden. Bisher konnten überwiegend stationär durchgeführte Asthmaschulungen ihre Effektivität belegen $[4,5]$. Jedoch können stationäre oder ambulante Gruppenschulungsprogramme häufig keine flächendeckende Versorgung gewährleisten. Ein großer Teil der Schulungsinhalte verliert im Alltag an Bedeutung und ist nach kurzer Zeit nicht mehr gegenwärtig [6]. Gruppenschulungsprogramme werden nach Schätzungen nur von ca. 30\% der Kinder, Jugendlichen und Familien wahrgenommen. Daher sind Nachschulungen dringend erforderlich. Nach den Vorgaben der AG Asthmaschulung und der Nationalen Versorgungsleitlinie ist die Durchführung einer Asthma-Nachschulung 6-12 Monate nach einer Basisschulung sinnvoll und notwendig [7,8]. Dabei zeigen mehrere Studien den Vorteil von internet- bzw. computerbasierten interaktiven Asthmaschulungsprogrammen gegenüber Standardschulungen [9-11]. Gerade die Affinität der Jugendlichen zum Internet bzw. zu Online-Spielen wird durch dieses Angebot genutzt.

Ziel dieser prospektiven Pilotstudie war es, den Einfluss einer vierwöchigen Nachschulung mittels des internetbasierten interaktiven Schulungsprogramms „Luftikids“ auf die Symptomatik, die Asthmakontrolle, die Krankheitsbewältigung und die Lungenfunktion zu untersuchen.

Bis dato gibt es keine Untersuchung, die ein internetbasiertes Asthma-Lernspiel für Kinder und Jugendliche im Alter von 8-14 Jahren evaluiert hat, das als Nachschulungsmaßnahme verordnet und dessen Kosten von Krankenkassen erstattet werden können. demonstrated. The results support the effectiveness and good acceptance of an outpatient, internet-based asthma refresher course. Young asthma patients in particular can succeed with and be motivated by this form of refresher course.

\section{Methodik \\ $\nabla$}

In unserer prospektiven Interventionsstudie wurden, im Rahmen von Verlaufskontrollen in unserer Asthmaambulanz, zunächst die Kinder und Jugendlichen bzw. Eltern über den Inhalt und Ablauf der Studie standardisiert aufgeklärt.

Folgende Einschlusskriterien wurden definiert: 1. Diagnose eines Asthma bronchiale basierend auf nationalen Kriterien, 2. Teilnahme an einer ambulanten/stationären Asthmaschulung in den letzten 6 Jahren, 3. Alter zwischen 8 und 14 Jahren, 4. Vorliegen der Einverständniserklärung des Patienten und eines Elternteils, 5. Internetanschluss am Wohnort. Ausschlusskriterien waren weitere chronische Krankheiten oder/und eine aktuelle Lungenerkrankung zum Zeitpunkt der Vorstellung.

Zu Beginn der Studie wurden der Basisfragebogen zur Erhebung der Patientendaten, Symptomatik und Medikation sowie der Fragenbogen zur Krankheitsbewältigung ausgefüllt und eine Lungenfunktionsuntersuchung durchgeführt. Bei der Angabe von Asthma-Auslösern bzw. Begleiterkrankungen waren Mehrfachnennungen möglich. Bei den Asthmasymptomen wurden die Variablen Husten, Dyspnoe und Asthmaanfälle mit den möglichen Angaben: „weniger als $1 \times$ pro 2 Monate, öfter als $1 \times$ pro 2 Monate, mehrmals wöchentlich und täglich“ erfragt. Außerdem wurde der Asthma Control Questionnaire (ACQ-7), bestehend aus 6 Fragen, orientierend an GINA-Kriterien, bezogen auf die letzte Woche, mit Berücksichtigung der Lungenfunktionsmessung $\left(\mathrm{FEV}_{1} \%\right)$ eingesetzt. Bei einem Skalenwert von $<0,75$ handelt es sich um ein gut kontrolliertes Asthma. Die Einschätzung des Asthmaschweregrads erfolgte zunächst durch die Patienten und wurde im Anschluss anhand der Therapiestufe festgelegt.

Ein Peak-Flow-Meter und ein Beobachtungsfragebogen wurden jedem Patienten für die nächsten 4-6 Monate mitgegeben. Danach erhielten die Patienten für 4 Wochen die Freischaltung des internetbasierten Programms „Luftikids“. Die Nutzer konnten selbst bestimmen, wie häufig und wie intensiv die Nachschulung erfolgte. Im Anschluss wurden 4-6 Monate nach Tag 1 der Studie die erneute Evaluation mittels der vorbeschriebenen Fragebögen und die zweite Lungenfunktionskontrolle in der Klinik durchgeführt $($ Abb. 1).

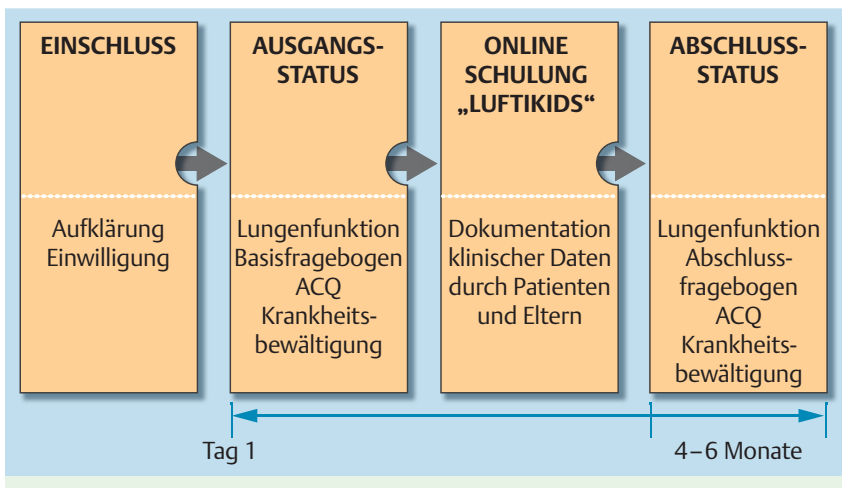

Abb.1 Studienablauf. 


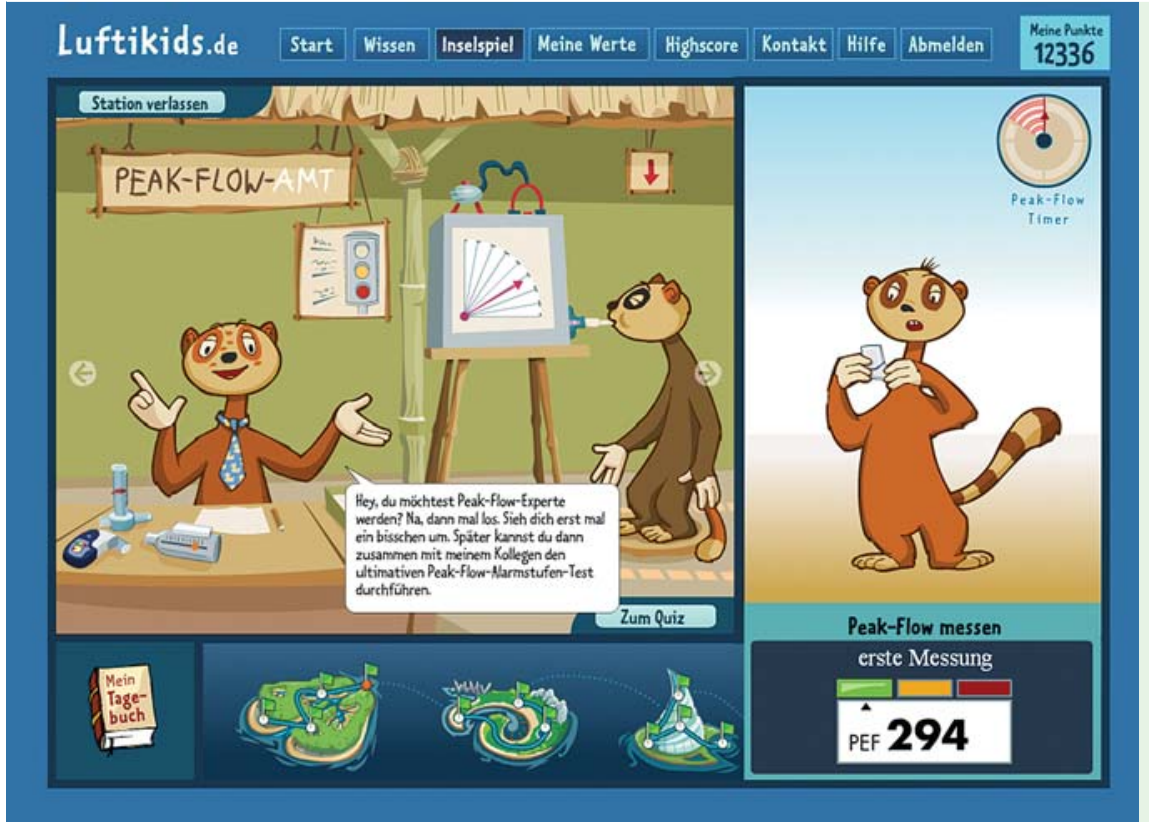

Abb.2 Beispiel für „Luftikids“ Inselspiel.

Untersucht wurde der Einfluss des internetbasierten Programms auf die Asthmasymptomatik (Husten, Dyspnoe, Anfall), die Anzahl ungeplanter Arztbesuche, den Gebrauch von Bedarfsmedikamenten ( $\beta$-Agonisten), den ACQ-Score und die Krankheitsbewältigung sowie die Lungenfunktionsparameter Vitalkapazität (VC), maximaler exspiratorischer Fluss (PEF), maximaler exspiratorische Fluss bei $50 \%\left(\mathrm{MEF}_{50}\right)$, Einsekundenkapazität $\left(\mathrm{FEV}_{1}\right)$ sowie Tiffeneau-Quotient $\left(\mathrm{FEV}_{1} / \mathrm{VC}\right)$. Weiterhin erfasst wurde die Nutzungsdauer der Online-Nachschulung in Minuten und die Einschätzung der Effektivität durch den Patienten.

Die Studie wurde durch die Ethikkommission des Fachbereichs Humanmedizin der Justus-Liebig-Universität Gießen genehmigt. Die Kinder und Jugendlichen sowie deren Eltern erhielten zu Studienbeginn ein Aufklärungsschreiben und nur nach Vorliegen der Einverständniserklärungen wurden die Patienten in die Studie eingeschlossen.

\section{Intervention}

„Luftikids“ ist ein Online-Lernspiel für Kinder und Jugendliche mit Asthma bronchiale. Die interaktive internetbasierte Lernanwendung wurde, auf Initiative von Dr. med. Behling, von Medienpädagogen, Mediendesignern und Programmierern der outermedia GmbH erarbeitet. Die medizinischen Inhalte wurden unter fachlicher Abstimmung und Kontrolle der AG Asthmaschulung erstellt und didaktisch auf die Bedürfnisse von Schulkindern und Jugendlichen im Alter von 8 bis 14 Jahren zugeschnitten. Die Anwendung steht dem Patienten jederzeit über das Internet zur Verfügung und kann mit jedem aktuellen Internet-Browser ausgeführt werden. Der Patient kann sich in den passwortgeschützten Bereich des Lernspiels einloggen, darin den Verlauf seines PeakFlows dokumentieren, in der Hauptnavigation des Lernspiels vorangehen und weitere Inhalte spielerisch erschließen.

Rudi, das Maskottchen, das, wie der Spieler auch, an Asthma leidet und damit die Identifikation des Spielers mit der Hauptfigur unterstützt, führt die Kinder durch eine interaktive Inselwelt mit Urbewohnern. Deren Idylle wird durch einen ausbrechenden Vulkan bedroht. Gemeinsam mit Rudi müssen die Aufgaben der Inselwelt gelöst und der Vulkanausbruch verhindert werden. Auf den fünf verschiedenen Inseln werden Themen wie Lunge und Atmung, Therapiemaßnahmen, Verhalten beim Asthmaanfall, Atem- und Entspannungsübungen, aktive Krankheitsbewältigung und die Notwendigkeit des Selbstmanagements vermittelt. Das angeeignete Wissen wird überprüft und bei erfolgreichem Abschluss wird der „drohende Vulkanausbruch“ mit resultierender Luftverunreinigung der Inselwelt verhindert. Neben dem Inselspiel ( $\mathbf{A}$ bb.2) mit 15 verschiedenen Einzelspielen steht zusätzlich ein umfangreicher Wissensbereich, aufgeteilt in thematische Kurse, zur Verfügung ( $\bullet$ Abb.3). Hier kann Wissen angeeignet, durch das Lösen von Quizfragen überprüft und bei Bedarf nachgeschlagen werden. Dieser Bereich ist auch von den Eltern gut nutzbar.

Zur Unterstützung der Selbstmanagementkompetenz bestimmt der Patient das Tempo und die Spielintensität selbst. Die OnlineSchulung steht für vier Wochen zur Verfügung und kann innerhalb dieses Zeitraums jederzeit genutzt werden. Durch eine vorgegebene Reihenfolge der Lektionen kann keine Aufgabe übersprungen werden. Dies führt dazu, dass der Patient an allen inhaltlichen Lektionen partizipiert. Die Anwendung beginnt nach dem Login mit der Abfrage des Peak-Flows und der Dokumentation möglicher Beschwerden. Eine Liste der erfassten Werte kann jederzeit vom Patienten abgerufen und ausgedruckt werden, um den Asthma-Verlauf (gemeinsam mit dem Arzt) auszuwerten. Durch das Lösen von Quizfragen, das Lesen von Wissensbereichen, das Dokumentieren eigener Peak-Flow-Werte sowie durch das Voranschreiten im Spiel erzielt der Patient Punkte, die ihm helfen, seine eigenen Spielerfolge zu bewerten. Zudem kann er sein Ergebnis mit den Punkten anderer Nutzer vergleichen. Ein solches High-Score-Element trägt zur anhaltenden Motivation der Spieler bei und erhöht gleichzeitig den Reiz, die Herausforderungen der 15 verschiedenen Spiele zu bewältigen und damit das Asthmawissen zu erhöhen.

Die Lernanwendung wurde mit mehreren Preisen ausgezeichnet (Deutscher Multimedia Award in der Rubrik "Interactive Services", "intermedia-globe silver" in der Kategorie "Webseiten/ eLearning" beim WorldMediaFestival, Siegertitel des KreativWettbewerbs “Annual Multimedia 2008”). 


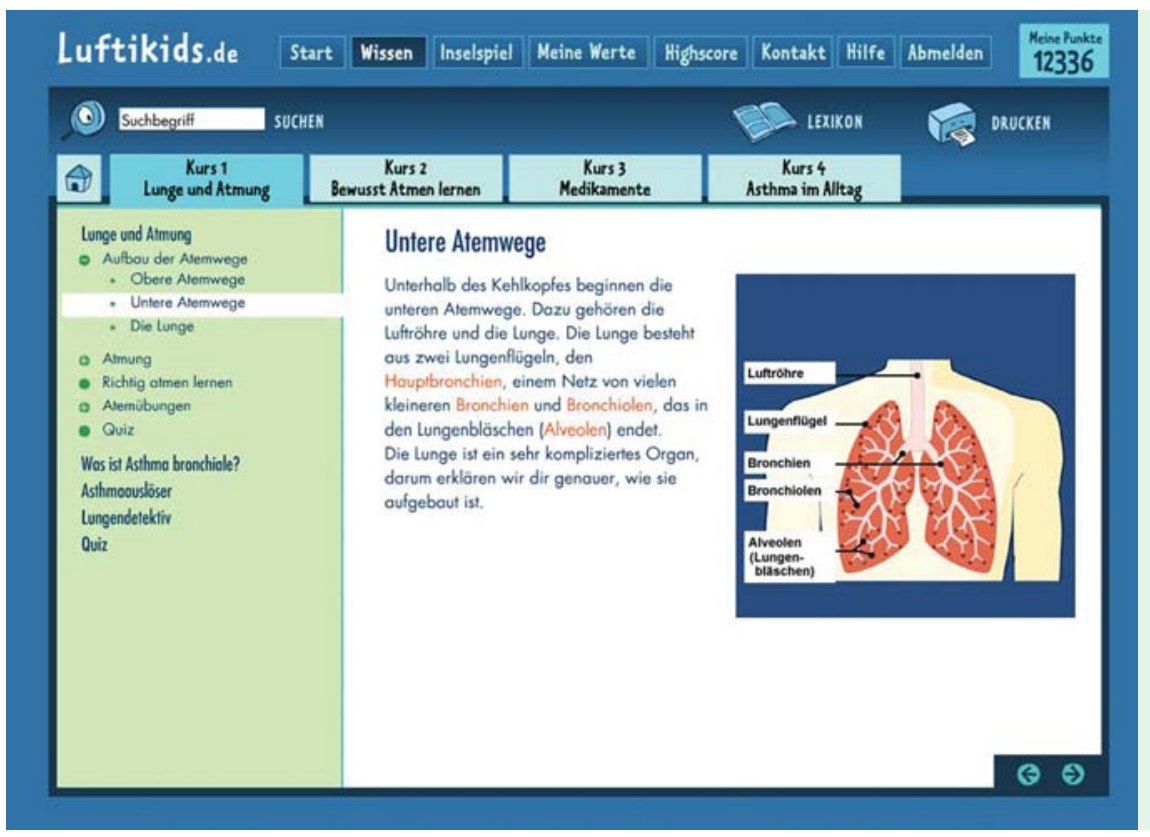

Abb.3 Beispiel für „Luftikids“ Wissensbereich.

\begin{tabular}{lcccc}
\hline & Anzahl Patienten (n) & Anzahl Patienten (\%) & Mittelwert & SD \\
\hline $\begin{array}{l}\text { Geschlecht } \\
\text { männlich }\end{array}$ & 35 & 66,0 & & \\
weiblich & 18 & 34,0 & 10,3 & 1,8 \\
\hline Alter & 53 & & & \\
\hline Asthmajahre & 53 & & \\
\hline Atopische Dermatitis & & & \\
ja & 14 & 26,4 & \\
nein & 37 & 69,8 & \\
k. A. & 2 & 3,8 & \\
Allergien & & & \\
ja & 36 & 67,9 & \\
nein & 15 & 28,3 & \\
k. A. & 2 & 3,8 & \\
Asthmaschweregrad & & & \\
intermittierend & 1 & 1,9 & \\
leichtgradig persistierend & 42 & 79,1 & \\
mittelgradig persistierend & 7 & 13,3 & \\
schwer persistierend & 0 & 0,0 & \\
k. A. & 3 & 5,7 & \\
\hline
\end{tabular}

Tab. 1 Klinische Charakteristika.

k. A.: keine Angaben

\section{Statistik}

Die statistische Datenanalyse erfolgte an der Universitäts-Kinderklinik Zürich. Bei normalverteilt stetigen Merkmalen kam für den Vergleich vor und nach der Intervention der gepaarte t-Test zur Anwendung. Die Resultate werden als Mean \pm Standard Deviation dargestellt. Bei nicht normalverteilten stetigen sowie bei ordinalskalierten Merkmalen wurde der Wilcoxon-Vorzeichen-Test verwendet. Das Signifikanzniveau wurde bei $5 \%$ festgelegt.

\section{Ergebnisse}

$\nabla$

Von 60 angefragten Patienten nahmen 54 Kinder und Jugendliche im Alter von 10,3 $\pm 1,8$ Jahren teil, welche bereits 6,6 $\pm 3,1$ Jahre an Asthma litten. Davon waren 35 Jungen (66\%) und 18 Mädchen (34\%). Ein Patient musste aufgrund eines akuten Atemwegsinfekts ausgeschlossen werden. Die meisten Probanden $(79,2 \%)$ hatten ein leichtgradiges Asthma. Kein Kind war an einem schweren persistierenden Asthma erkrankt. Von den Probanden waren $67,9 \%$ Allergiker, und $26,4 \%$ hatten eine Neurodermitis ( Tab.1).

Als Medikation verwendeten 92,5\% der Patienten inhalative Steroide in einer Tagesdosis von 100-400 $\mu$ g (BDP-Äquivalenz) als Dauertherapie. Weitere häufig verwendete Substanzen zur Dauermedikation waren kurzwirksame $\beta_{2}$-Mimetika (56,6\%) und Leukotrienantagonisten (22,6\%). Langwirksame $\beta_{2}$-Mimetika, Parasympatholytika, Theophyllin, systemische Steroide wurden nur sehr selten oder nie benutzt. Eine Bedarfsmedikation (SABA) war bei allen Patienten (100\%) vorhanden.

Eine Basisschulung hatten bereits 52 Kinder und Jugendliche (98,1\%) absolviert, bei einem Patienten fehlte die diesbezügliche Angabe. Alle 53 Patienten gaben an, eine Nachschulung besucht zu haben. Bei zwei Drittel (66\%) war die Nachschulung individuell im Rahmen der Vorstellung in unserer Ambulanz erfolgt, bei einem Drittel wurden verschiedene Nachschulungsarten genutzt. Gruppenschulung oder Rehabilitationsaufenthalte wurden nie 
oder nur sehr selten als einzige Nachschulungsart genannt. Das Asthma bronchiale der Patienten war bereits bei Eintritt in die Studie meist sehr gut kontrolliert: Der ACQ-7 Score betrug bei Einschluss $0,6 \pm 0,7$ (mean $\pm S D$ ).

Die Kinder und Jugendlichen beschäftigten sich während der 4-wöchigen Intervention 302,0 $\pm 210,9$ Minuten mit dem Nachschulungsprogramm „Luftikids“. Die Anzahl der Logins betrug $14 \pm 9$ (4-36). 30,2\% der befragten Patienten schätzten ihre Spieldauer auf bis zu $1 \mathrm{~h}$ pro Tag ( $\bullet$ Abb.4). Eigeneinschätzung und absolute Spieldauer waren signifikant korreliert $(p=0,002)$. Das Spielende nach Abschluss der 5. Insel wurde von $24,5 \%$ erreicht, 71,7\% gelangten zur Insel 3 und 4 $\pm 1,2$. Der Highscore betrug $4870 \pm 3287,1(240-11316)$. Von den 14 Quiz wurden $8 \pm 5$ $(0-14)$ bestanden.

Die meisten Patienten profitierten gemäß eigener Einschätzung viel bis sehr viel. In einer Likert-Skala, welche in 5 gleichgroße Abschnitte geteilt wurde ( 1 bis 5 , kein Nutzen bis sehr viel Nutzen), lagen $56,6 \%$ in den Bereichen 4 und 5 ( $\bullet$ Abb.5). Die Einschätzung korrelierte mit der Spieldauer $(r=0,406, p=0,004)$, dem Highscore $(r=0,368, p=0,009)$ und der Anzahl erfolgreich absolvierter Quiz ( $r=0,312, p=0,029)$.

Eine Änderung der Lungenfunktionsparameter und des ACQScores vor und nach der Intervention konnte nicht nachgewiesen werden ( $\bullet$ Tab.2). Die Intensität der Beschwerdesymptomatik Husten und Atemnot verminderte sich jedoch signifikant $(\mathrm{p}=0,001$ resp. $\mathrm{p}=0,007 ; \bullet$ Abb. 6 und $\bullet$ Abb. 7). Auch ungeplante Arztbesuche $(p=0,005)$, der Einsatz der Bedarfsmedikation und Fehltage in der Schule reduzierten sich nach der internetbasierten Schulung ( $\bullet$ Tab.2). Weiter verbesserten sich Asthmawissen und Asthmakontrolle; 76,6\% schätzten am Ende der Studie ihr Wissen als gut oder sehr gut ein, zu Beginn waren es 35,9\% $(p<0,001)$. Betreffend der „gefühlten“ Asthmakontrolle schätzten sich anfangs $34 \%$ der Kinder als sehr gut kontrolliert ein, bei Studienende waren es $52,8 \%(p=0,028)$.

\section{Diskussion}

$\nabla$

Die Nachschulung wird seit langem postuliert und ist für den Langzeiterfolg einer Asthmaschulung essenziell $[4,5,12,13]$. So konnten Sundberg et al. bei jungen Asthmapatienten (18 - 25 Jahre) zeigen, dass zeitlich begrenzte Aufklärungsprogramme über Krankheitszusammenhänge, ohne eine entsprechende Nachschulung, nur mäßige Auswirkungen auf klinisch messbare

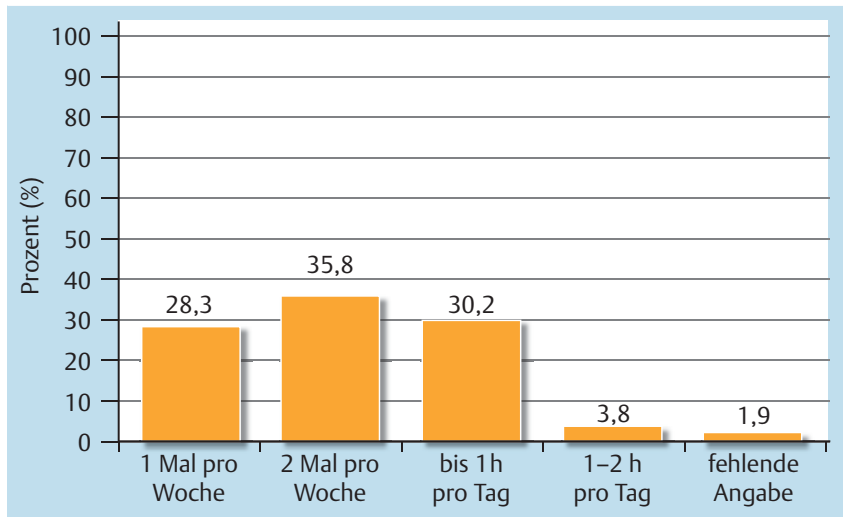

Abb.4 Einschätzung der Spielnutzung durch Patienten.

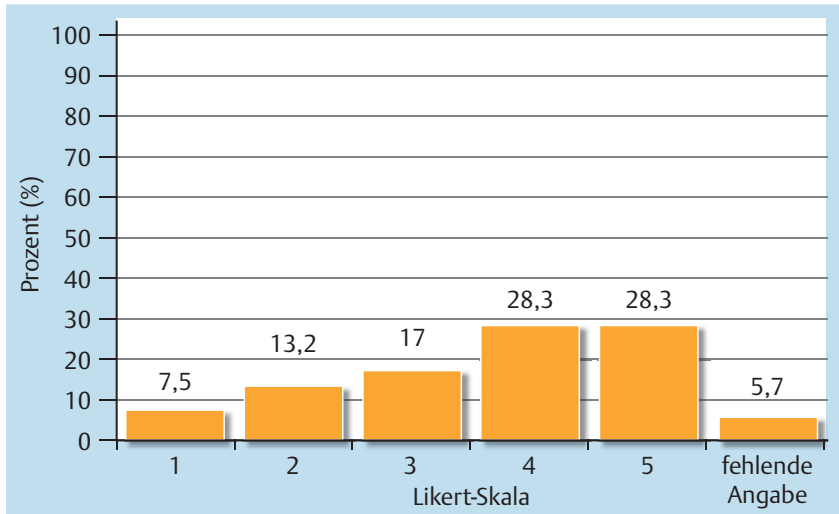

Abb.5 Einschätzung des eigenen Profits durch Patienten (1 = kein Nutzen, $5=$ sehr viel Nutzen).

Krankheitssymptome, asthmaspezifisches Fachwissen oder Lebensqualität haben [14]. Aufgrund struktureller Probleme mit fehlenden ambulanten Schulungsmöglichkeiten im direkten Umfeld sowie häufig mangelnder Motivation der Patienten wird die Form der konventionellen Nachschulung jedoch wenig genutzt. Eine internetbasierte Nachschulung zielt darauf ab, möglichst viele Patienten zu erreichen, und steht unabhängig von räumlichen oder zeitlichen Vorgaben zur Verfügung. Auf jugendgerechte Weise sollen Selbstwahrnehmung, soziale Kompetenz und das Wissen über die eigene Erkrankung vertieft werden.

Tab. 2 Veränderungen weiterer Symptome und Parameter vor und nach der internetbasierten Asthma-Nachschulung.

\begin{tabular}{|c|c|c|c|}
\hline & Vorher & Nachher & p-Wert \\
\hline \multicolumn{4}{|l|}{ Asthmakontrolle } \\
\hline $\begin{array}{l}\text { ungeplante Arztbesuche letzte } \\
4 \text { Wochen }\end{array}$ & 13 Patienten $(24,5 \%)$ & 2 Patienten $(3,8 \%)$ & $p=0,005$ \\
\hline $\begin{array}{l}\text { Bedarfsmedikation } \\
\text { letzte } 4 \text { Wochen }\end{array}$ & $4,0 \pm 6,5(0-38)$ & $1,5 \pm 4,9(0-28)$ & $p=0,002$ \\
\hline Fehltage Schule & $1,2 \pm 2,0(0-10)$ & $0,4 \pm 1,2(0-7)$ & $p=0,003$ \\
\hline ACQ-7 & $0,6 \pm 0,7(0-3,9)$ & $0,6 \pm 0,6(0-2,3)$ & $p=0,244$ \\
\hline \multicolumn{4}{|l|}{ Lungenfunktionsparameter } \\
\hline VC [I] & $2,6 \pm 0,6(1,9-4,6)$ & $2,7 \pm 0,6(2,0-4,7)$ & $p=0,350$ \\
\hline $\mathrm{FEV}_{1}[\mathrm{I}]$ & $95,0 \pm 12,0(60,0-118,0)$ & $95,3 \pm 12,6(51,0-112,0)$ & $p=0,346$ \\
\hline $\mathrm{FEV}_{1} / \mathrm{VC}[\%]$ & $81,0 \pm 8,0(59,1-93,9)$ & $81,0 \pm 6,3(51,0-118,0)$ & $p=0,346$ \\
\hline PEF [\%] & $87,2 \pm 14,7(53,0-115,0)$ & $94,5 \pm 15,2(60,0-128,0)$ & $p=0,145$ \\
\hline $\mathrm{MEF}_{50}[\%]$ & $75,1 \pm 19,1(37,0-102,0)$ & $74,7 \pm 17,8(29,0-102,0)$ & $p=0,258$ \\
\hline
\end{tabular}




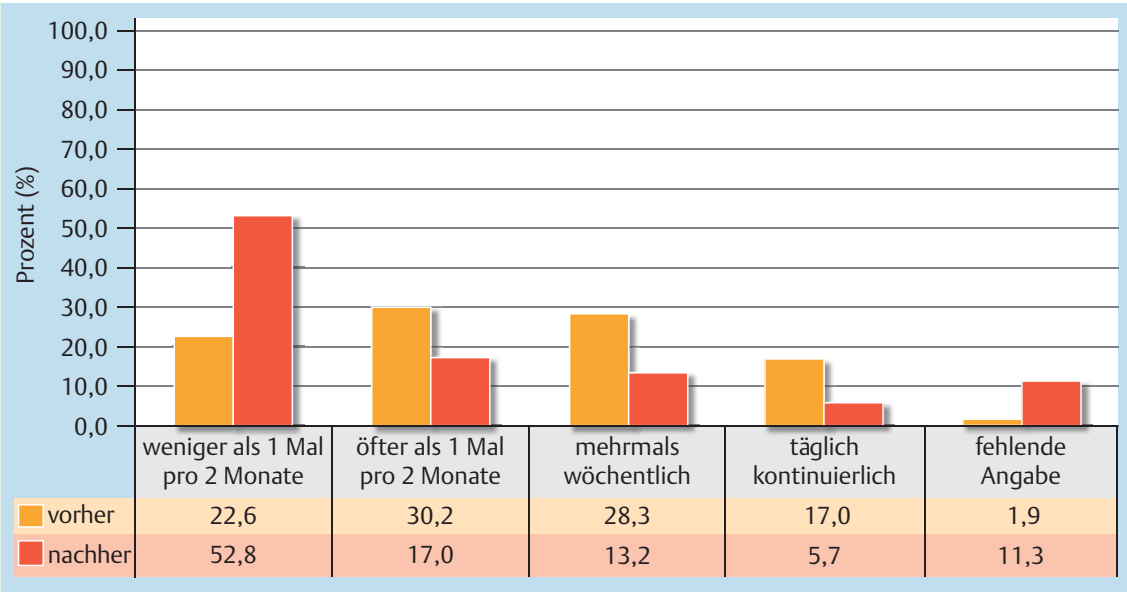

Abb. 6 Hustensymptomatik vor und nach Intervention $(p=0,001)$

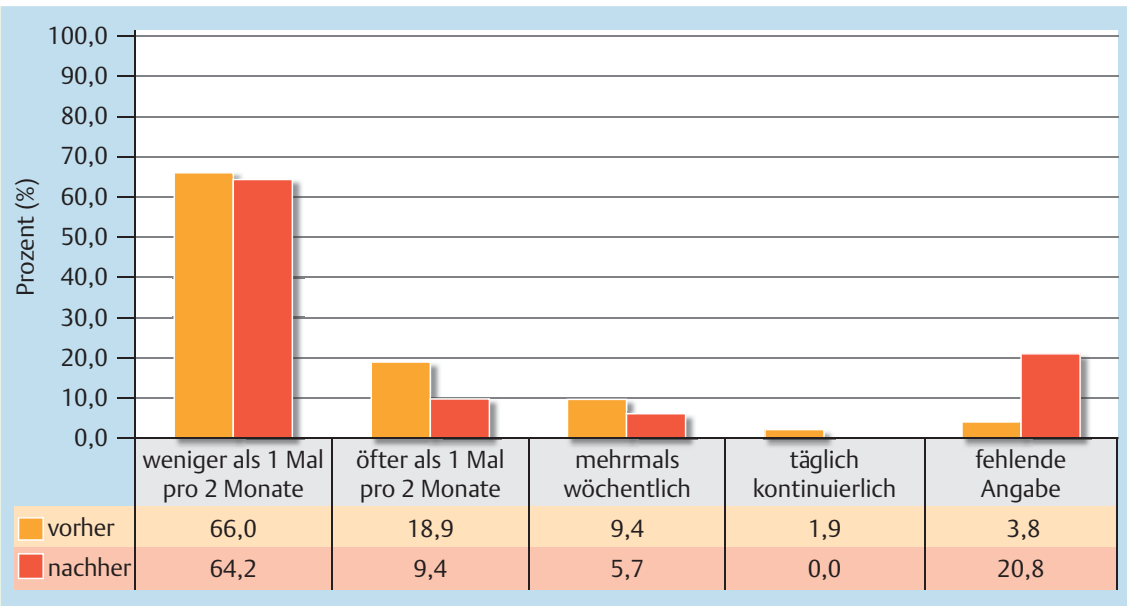

Abb.7 Atemnotsymptomatik vor und nach Intervention $(p=0,007)$.

Es geht dabei insbesondere um die Auffrischung des Gelernten; dies wird spielerisch vermittelt in Kombination mit Quizfragen und Spielen. Die Selbstwahrnehmung wird unter Hinzunahme des Peak-Flow-Meters geschult. Wir konnten in vergleichbarer Größenordnung wie andere Studien [11] signifikante Effekte mit einer verminderten Intensität der Asthmasymptomatik, einer Reduktion ungeplanter Arztbesuche, einer Reduktion des Gebrauchs der Bedarfsmedikation, einer Verminderung der Schulfehltage sowie eine bessere Selbsteinschätzung der Asthmakontrolle aufzeigen. Als wichtige Limitation ist anzumerken, dass auf eine Kontrollgruppe in unserer Pilotstudie verzichtet wurde, da uns eine zeitgemäße Therapie ohne Schulungskomponenten nicht vertretbar erschien. Anhand randomisierter und kontrollierter Studien mit größeren Fallzahlen sollten unsere Ergebnisse validiert werden. Nachteilig ist bei dieser Form der Schulung, dass keine Erfahrungen mit anderen Betroffenen ausgetauscht werden können [15].

Voraussetzung einer erfolgreichen internetbasierten Asthmanachschulung ist die Möglichkeit eines flächendeckenden Einsatzes, die Begleitung durch einen professionellen Anbieter sowie die inhaltliche und strukturelle Erfüllung von Vorgaben der Fachgesellschaften gemäß den Asthma-Leitlinien [8].

Der Vergleich mit anderen Studien bezüglich der Effektivität von multimedialen Asthmaschulungsprogrammen im Kindes- und Jugendalter ergab in randomisiert-kontrollierten Studien eine Reduktion von Symptomen, Anzahl der Hospitalisationen und eine Wissenszunahme des Asthmaselbstmanagements im Vergleich zu den Kontrollgruppen $[9,16,17]$.
Chan et al. und Homer et al. konnten Verbesserungen in der Krankheitsbewältigung aufzeigen, diese zeigten jedoch keinen Unterschied zu den Kontrollen, welche andere Formen einer Asthmaschulung erhielten $[10,18]$. Von einer Verbesserung der Lungenfunktionsparameter konnten lediglich Jan et al. bezüglich des PEF berichten [16]. In allen anderen zitierten Arbeiten waren die Lungenfunktionsparameter vor und nach Intervention unverändert. Wir konnten zu Beginn unserer Untersuchung mittels des ACQ-Scores aufzeigen, dass das Asthma bei den Patienten bereits sehr gut medikamentös kontrolliert war. Darauf weisen ein PEF von $87,2 \%$ bzw. ein FEV $_{1}$ von $95 \%$ hin. Zudem litt kein Patient an einer schweren Asthmaform, und über $90 \%$ waren bereits mit inhalativen Steroiden therapiert. Die guten Ausgangswerte der Lungenfunktion weisen darauf hin, dass es sich bei der Studienpopulation um eine vorselektionierte Gruppe handelt, die, wie erwähnt, bereits mindestens eine Asthmagrundschulung erhalten hatte.

Trotz dieser Einschränkung ist es umso erstaunlicher, dass sich nach der Intervention die Variablen Husten, Dyspnoe und Gebrauch der Bedarfsmedikation sowie das Asthmawissen signifikant verbesserten und mit Highscore und Anzahl bestandener Quiz korrelierte; zumal der ACQ-7 sich nicht änderte.

Obwohl bereits eine gute Asthmakontrolle, bewertet durch den validierten ACQ-Score und verschiedene Lungenfunktionsparameter, bestand, schätzten die Patienten die subjektive Intensität der Beschwerden anfangs relativ hoch ein. Nach der internetbasierten Nachschulung wurde die Intensität tiefer bewertet. Diese Beobachtung könnte durch ein verbessertes Asthmawissen 
bedingt sein; die Patienten schätzten ihre Krankheit nun realistischer ein, wodurch sich subjektive und objektive Kriterien der Asthmakontrolle annähern. Jedoch ist auch ein direkter Einfluss von „Luftikids“ möglich. Die Kinder und Jugendlichen haben Atem- und Entspannungsübungen sowie den adäquaten Einsatz der Bedarfsmedikation erlernt.

Die zitierten Studien unterscheiden sich sehr im Hinblick auf Art und Inhalt der multimedialen Schulungsprogramme. Teilweise waren die Zugänge zu den Programmen ausschließlich auf die Arztbesuche beschränkt $[9,17,18]$. Die Schulungen dienten überwiegend der Erstschulung. Der Effekt einer Nachschulung wurde nur von Runge et al. und Krishna et al. untersucht $[9,11]$. Keine Studie untersuchte - wie von uns evaluiert - die Häufigkeit und Gesamtdauer der Nutzung.

Unsere Daten der Pilotstudie zeigen, dass mittels eines internetbasierten Nachschulungsprogramms die Versorgung von Kindern und Jugendlichen mit Asthma bronchiale verbessert werden kann. Insbesondere die flächendeckende Implementierung des Nachschulungsprogramms ist gewährleistet. Sowohl die hohe Profiteinschätzung als auch die durchschnittliche Spieldauer und Regelmäßigkeit des Logins sprechen für die gute Akzeptanz durch die Nutzer. Die internetbasierte Schulung „Luftikids“ stellt damit eine effektive und praktikable Variante der Asthmaedukation im Kindes- und Jugendalter dar.

\section{Interessenkonflikt}

$\nabla$

Die Autoren geben an, dass kein Interessenkonflikt besteht.

\section{Literatur}

1 Trzcieniecka-Green A, Bargiel-Matusiewicz K, Wilczynska-Kwiatek A. Quality of life and activity of children suffering from bronchial asthma. Eur J Med Res 2009 (Suppl. 14): 147-150

2 Kohen DE. Asthma and school functioning. Health Rep 2010; 21: 35 45

3 Guevara JP, Wolf FM, Grum CM et al. Effects of educational interventions for self management of asthma in children and adolescents: systematic review and meta-analysis. BMJ 2003; 326: 1 -6
4 Bauer CP, Petermann F, Kiosz D et al. Langzeiteffekt der stationären Rehabilitation bei Kindern und Jugendlichen mit mittelschwerem und schwerem Asthma bronchiale. Pneumologie 2002; 56: 478 - 485

5 Gebert N, Hümmelink R, Könning J et al. Efficacy of a self-management program for childhood asthma - a prospective controlled study. Patient Educ Couns 1998; 35: $213-220$

6 SteißJO, Schudt J, Landmann E et al. Zur Effizienz der Nachschulung von Kindern und Jugendlichen mit Asthma bronchiale. Allergologie 2006; 29: $109-116$

7 Arbeitsgemeinschaft Asthmaschulung im Kindes- und Jugendalter Qualitätsmanagement in der Asthmaschulung von Kindern und Jugendlichen. 3. Aufl., Zuckerschwerdt; 2007

8 Nationale Versorgungsleitlinie Asthma. 2. Aufl. (aktualisiert 11/2010), Dtsch Ärztebl 20114: 192 -195. Verfügbar in www.versorgungsleitlinien.de

9 Krishna S, Francisco BD, Balas EA et al. Internet-enabled interactive multimedia asthma education program: a randomized trial. Pediatrics 2003; 111: 503-510

10 Chan DS, Callahan CW, Hatch-Pigott VB et al. Internet-based home monitoring and education of children with asthma is comparable to ideal office-based care: results of a 1-year asthma in-home monitoring trial. Pediatrics 2007; 119: 569-578

11 Runge C, Lecheler J, Horn $M$ et al. Outcomes of a web-based patient education program for asthmatic children and adolescents. Chest United States 2006: $581-593$

12 Kiosz D, Szcepanski R, Brockmann G et al. Ambulante Nachschulung verbessert den Effekt stationärer Asthmaschulung im Kindesalter. Präv Rehab 2001; 13: 93-104

13 Szczepanski R, Brockmann G, Friede G. Asthmaschulung für Lehrer Notwendigkeit und Möglichkeiten. Pneumologie 2001; 55: 512 - 519

14 Sundberg R, Tunsäter A, Palmqvist $M$ et al. A randomized controlled study of a computerized limites education program among young adults with asthma. Respir Med 2005; 99: 321 - 328

15 Wilson S. Individual versus group education: Is one better? Patient Educ Cours 1997; 32: 67-75

16 Jan RL, Wang JY, Huamng MC et al. An internet-based interactive telemonitoring system for improving childhood asthma outcomes in Taiwan. Telemed J E Health 2007; 13: 257-268

17 Bartholomew LK, Gold RS, Parcel GS et al. Watch, Discover, Think, and Act: evaluation of computer-assisted instruction to improve asthma self-management in inner-city children. Patient Educ Couns 2000; 39: $269-280$

18 Homer C, Susskind O, Alpert HR et al. An evaluation of an innovative multimedia educational software program for asthma management: report of a randomized, controlled trial. Pediatrics 2000; 106: $210-$ 215 